Extra View

\title{
O-linked $\beta$-N-acetylglucosamine during hyperglycemia exerts both anti-inflammatory and pro-oxidative properties in the endothelial system
}

\author{
Angana Gupta Rajapakse, Xiu-Fen Ming, João M. Carvas and Zhihong Yang* \\ Vascular Biology; Department of Medicine; Division of Physiology; Faculty of Science; University of Fribourg; Fribourg, Switzerland
}

Key words: adhesion molecules, endothelial cells, glucosamine, inflammation, ROS

Elevated cellular levels of protein $O$-linked $\beta$-N-acetylglucosamine $(O-G l c N A c)$ through hexosamine biosynthesis pathway (HBP) are suggested to contribute to cardiovascular adverse effects under chronic hyperglycemic condition associated with oxidative stress and inflammation. Conversely, enhancing $O$-GlcNAc levels have also been demonstrated being protective against myocardial ischemia/reperfusion injury. We recently demonstrated that hyperglycemia increases oxidative stress and HBP flux in endothelial cells and enhances endothelial expression of vascular adhesion molecule-1 (VCAM-1) and intercellular adhesion molecule-1 (ICAM-1) in response to tumor necrosis factor- $\alpha(\mathrm{TNF} \alpha)$ through oxidative stress rather than HBP pathway. Here we present further complementary data showing that enhancing $O$-GlcNAc levels by glucosamine does not mimic hyperglycemia's effect on TNF $\alpha$-induced endothelial VCAM-1 and ICAM-1 expression. Glucosamine however inhibits ICAM-1 (not VCAM-1) expression and induces superoxide generation in the cells. The results further suggest that increased $O$-GlcNAc levels do not mediate the enhancing effect of hyperglycemia on the endothelial inflammatory responses to TNF $\alpha$. In contrast, it exerts certain anti-inflammatory effects accompanied by prooxidative properties. Further work should delineate the exact role of HPB pathway in different aspects of cardiovascular functions, especially those of diabetic cardiovascular complications.

\section{Introduction}

The posttranslational modification of serine and threonine residues of proteins by the $O$-linked attachment of the monosaccharide $\beta$ - $\mathrm{N}$-acetylglucosamine (O-GlcNAc), i.e.,

${ }^{*}$ Correspondence to: Zhihong Yang; Laboratory of Vascular Biology; Department of Medicine; Division of Physiology; University of Fribourg; Rue du Musée 5; Fribourg CH-1700 Switzerland; Tel.: +0041.26.300.85.93; Fax: +0041.26.300.97.34; Email: zhihong.yang@unifr.ch

Submitted: 03/13/09; Revised: 03/18/09; Accepted: 03/18/09

Previously published online as an Oxidative Medicine and Cellular Longevity E-publication:

http://www.landesbioscience.com/journals/oximed/article/8482 protein $O$-GlcNAcylation is emerging to play critical roles in regulation of wide spectrum of cellular functions including gene transcription, protein translation, proteasomal degradation and signal transduction. ${ }^{1}$ An elevated cellular level of $O$-GlcNAc is best described under chronic hyperglycemic condition in which an increased portion of glucose entering the cell is shunted from glycolysis to the hexosamine biosynthesis pathway (HBP). ${ }^{1}$ In the HBP pathway, glucose is converted to glucosamine-6-phosphate by the rate-limiting enzyme, L-glutamine:D-fructose-6-phosphate amidotransferase (GFAT). Glucosamine-6-phosphate is further metabolized to UDP- $N$ acetylglucosamine (UDP-GlcNAc) that serves as the major substrate for the formation of $O$-GlcNAc-modified proteins at serine and threonine residues catalysed by the UDP-GlcNAc:polypeptide $O-\beta$-N-acetyl-glucosaminyltransferase (OGT). ${ }^{1}$ It has been suggested that elevated $O$-GlcNAc contributes to many deleterious effects of hyperglycemia, i.e., glucotoxicity including insulin resistance, diabetic cardiovascular damage associated with oxidative stress and inflammation. ${ }^{1-3}$

Conversely, there is a growing body of data demonstrating that enhancing $O$-GlcNAc level is protective against cellular injury, improves cell survival and reduces ischemia/reperfusion injury and myocardial infarction in animal models. ${ }^{4,5}$ The cellular $O$-GlcNAc levels are augmented under the acute non-diabetic stress condition i.e., ischemic preconditioning in the heart. ${ }^{6,7} \mathrm{An}$ increase in $O$-GlcNAc level can be induced pharmacologically with the addition of exogenous glucosamine, which enters cells via the glucose transporter system and is phosphorylated to glucosamine-6phosphate by hexokinase, thus bypassing GFAT and leading to a rapid increase in UDP-GlcNAc levels. ${ }^{8}$ Glucosamine has been shown to possess anti-inflammatory properties in variety of disease models including rheumatoid arthritis, cardiac allograft survival, balloon angioplasty induced arterial injury and cardiac ischemia/ reperfusion injury. ${ }^{9-11}$ The mechanisms of the apparent paradox of the cellular protective and deleterious effects of increased $O$-GlcNAc levels are not known. It is also not clear what is the functional role of the increased $O$-GlcNAc under the hyperglycemic condition in cardiovascular system. 
In our most recently published article, ${ }^{12}$ we showed that in cultured human umbilical vein endothelial cells (HUVEC) hyperglycemia increases oxidative stress and HBP flux, amplifies endothelial inflammatory responses i.e., VCAM-1 and ICAM-1 expression in response to TNF $\alpha$, and impairs endothelium-dependent relaxations in isolated blood vessels mainly through oxidative stress rather than through HBP pathway. In the current article we present further complementary results showing functional roles of increased $O$-GlcNAc levels in regulation of endothelial inflammatory responses and oxidative stress.

\section{Results}

Our recent study ${ }^{12}$ showed that hyperglycemia enhances endothelial expression of VCAM-1 and ICAM-1 in response to TNF $\alpha$ through increased oxidative stress rather than through increased cellular $O$-GlcNAc levels. In the current study, we further investigated whether treatment of the cells with glucosamine to enhance $O$-GlcNAc levels could mimic the hyperglycemia's effect. In contrast to hyperglycemia, ${ }^{12}$ incubation of the cells with glucosamine $(0.1$ and $0.5 \mathrm{mmol} / \mathrm{L})$ for five days did not enhance TNF $\alpha$ ( $1 \mathrm{ng} / \mathrm{ml}, 24$ hours) induced VCAM-1 and ICAM-1 protein levels, but inhibited ICAM-1 (not VCAM-1) expression (Fig. 1). The results further support our conclusion that increased $O$-GlcNAc does not contribute to the hyperglycemiainduced upregulation of endothelial expression of the adhesion molecules in response to TNF $\alpha$. Of note, glucosamine itself had no significant effects on the basal level of endothelial expression of the adhesion molecules (Fig. 1). In that recent study, we also showed that hyperglycemia further enhances oxidative stress in response to $\mathrm{TNF} \alpha,{ }^{12}$ this interaction between high glucose and TNF $\alpha$ was however not observed between glucosamine and TNF $\alpha$ (Fig. 2). Although incubation of the cells with glucosamine alone (0.5 mol/L, 5 days) stimulated superoxide generation (similar to the high glucose condition ${ }^{12}$ ), it however did not significantly affect TNF $\alpha$ ( $1 \mathrm{ng} / \mathrm{ml}, 10$ minutes)-induced superoxide anion production as demonstrated by the dihydroethidium (DHE) fluorescence staining in the endothelial cells (Fig. 2), suggesting pro-oxidative properties of the HBP flux in endothelial cells.

\section{Discussion}

Protein $O$-GlcNAc modification has been recently shown to play important roles in regulation of variety of cellular functions. ${ }^{1}$ Under chronic hyperglycemic conditions of in vitro and in vivo model systems, increased HBP flux in different tissue/cells has been implicated in pathogenesis of insulin resistance and diabetic cardiovascular complications. ${ }^{13,14}$ For example, both high glucose and glucosamine increases $O$-GlcNAc modification of Akt/eNOS, leading to decreased activation of Akt/eNOS, ${ }^{15-17}$ an important vascular protective mechanism exerted by the vascular endothelial cells. ${ }^{18}$ Moreover, it has been demonstrated that elevation of $O$-GlcNAc levels by high glucose, glucosamine and overexpression of GFAT enhances plasminogen activator inhibitor-1 (PAI-1) expression in mesangial cells via activation of $\mathrm{Sp} 1$ site. ${ }^{19}$ Increasing $O$-GlcNAc by glucosamine also reduces vascular sprouting in mouse aortic rings and in aortas from diabetic mouse models. ${ }^{20}$

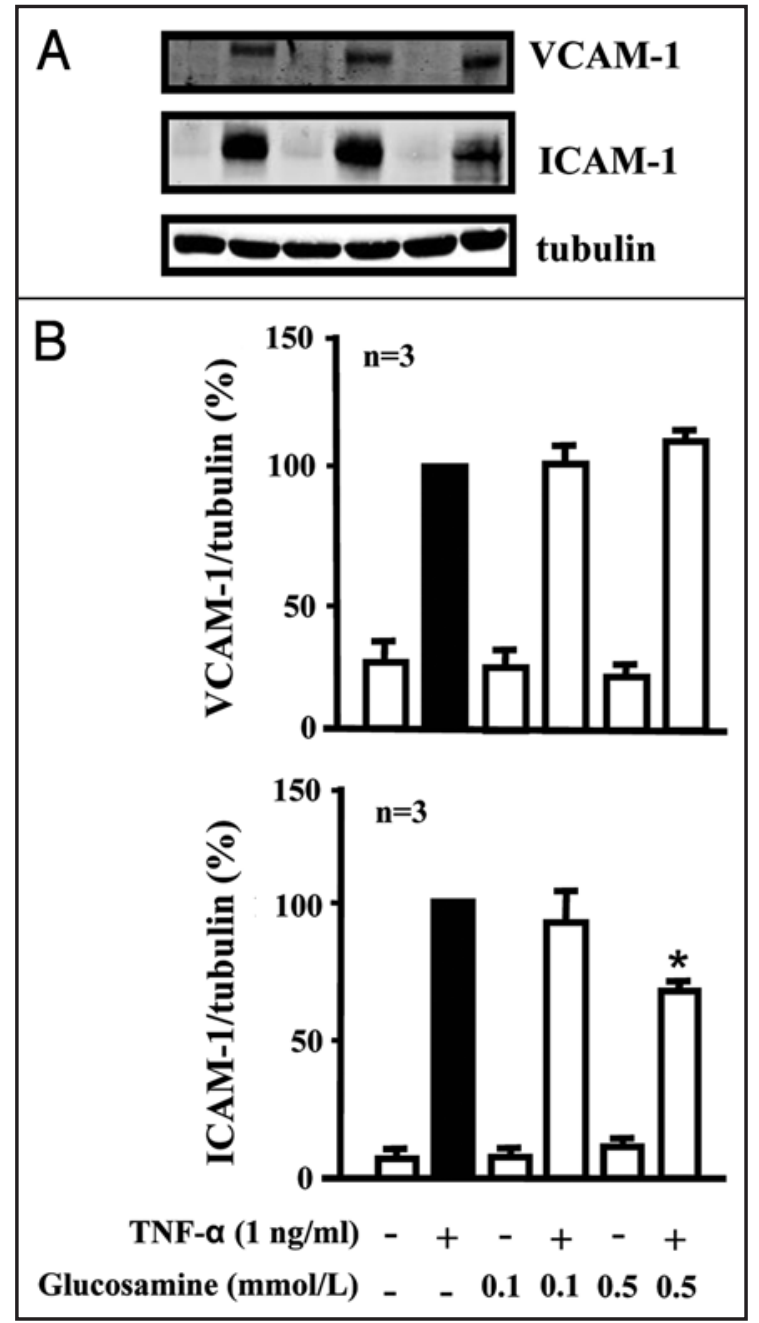

Figure 1. Effects of glucosamine on endothelial VCAM-1 and ICAM-1 expression. (A) Immunoblotting demonstrates that treatment of HUVECs with glucosamine $(0.1$ or $0.5 \mathrm{mmol} / \mathrm{L}, 5$ days) had no effects on VCAM-1, but reduced ICAM-1 protein expression in response to TNF $\alpha(1 \mathrm{ng} / \mathrm{ml}$, 24 hours, $n=3$ ). (B) Quantification of the above results. ${ }^{*} p<0.05$ vs. $\mathrm{TNF} \alpha$ alone.

All the studies implicate that increased HBP flux plays a role in the pathogenesis of diabetic cardiovascular complications.

The biological functions of HBP pathway in cardiovascular disease are recently challenged by a growing number of studies demonstrating that elevation of $O$-GlcNAc levels protects cardiomyocytes from ischemia/reperfusion injury and reduces myocardial infarction in animal models. ${ }^{4-7,9}$ The cellular $O$-GlcNAc levels are transiently augmented under the acute non-diabetic stress condition i.e., ischemic preconditioning in the heart. ${ }^{6,7}$ Augmentation of $O$-GlcNAc with high glucose or glucosamine improves cell survival, whereas decrease in $O$-GlcNAc levels reduces cell viability. ${ }^{4-7,9}$

In our most recently published article, ${ }^{12}$ we investigated the roles of oxidative stress and HBP pathway in hyperglycemiainduced endothelial inflammatory responses and endothelial dysfunction. We provided evidence that hyperglycemia increases oxidative stress and HBP flux in endothelial cells and enhances 


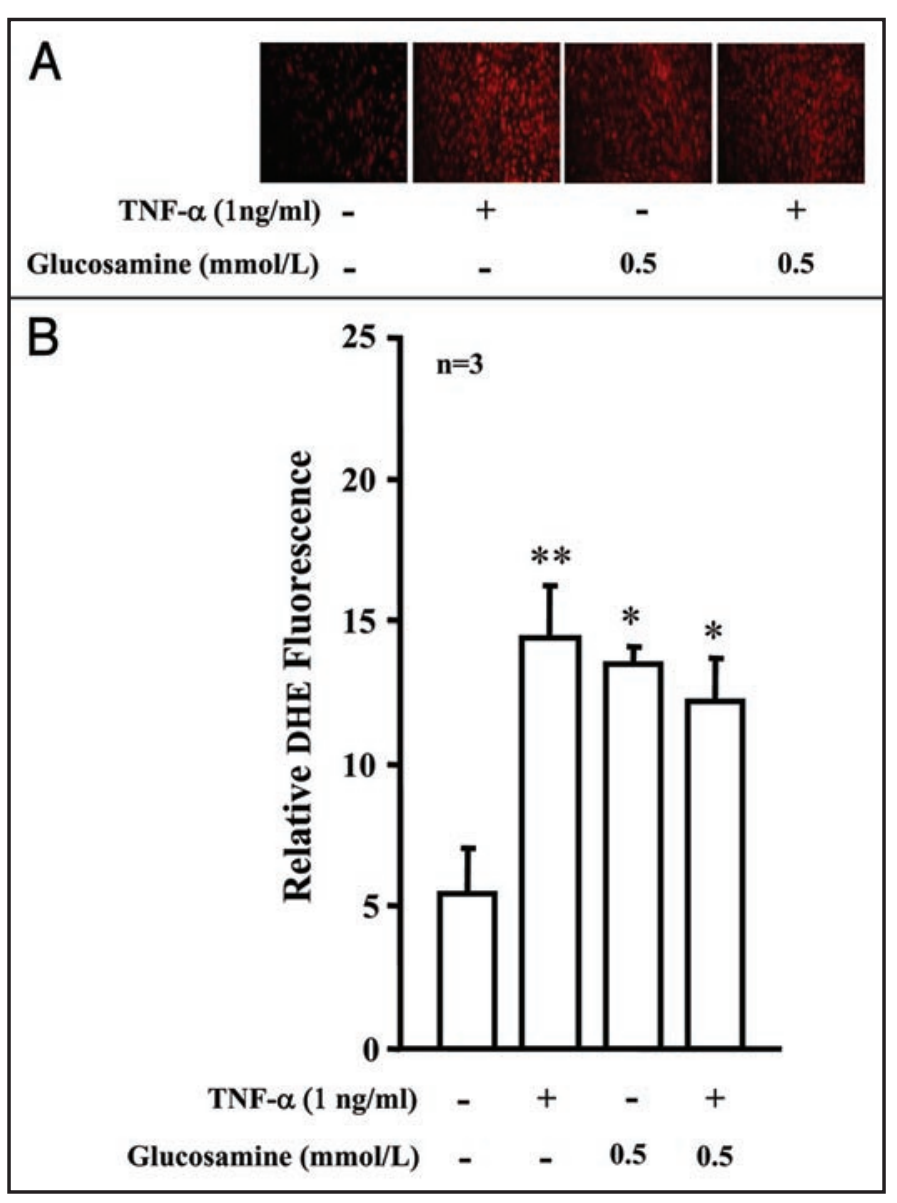

Figure 2. Effects of glucosamine on endothelial superoxide anion generation. (A) Dihydroethidium (DHE) fluorescence staining shows that glucosamine itself $(0.5 \mathrm{mmol} / \mathrm{L}, 5$ days) induced superoxide generation in HUVECs and did not affect the effect of TNF $\alpha(1 \mathrm{ng} / \mathrm{ml}, 10$ minutes, $\mathrm{n}$ =3). (B) Quantification of the above results. ${ }^{*} p<0.05$ and ${ }^{*} p<0.01$ vs. control.

endothelial inflammatory responses i.e., VCAM-1 and ICAM-1 expression in response to TNF $\alpha$ through oxidative stress rather than through HBP pathway, since inhibition of superoxide generation by resveratrol prevents the endothelial expression of the adhesion molecules stimulated by high glucose plus TNF $\alpha$ without inhibition of the global $O$-GlcNAc levels in the cells exposed to hyperglycemia, although alterations of $O$-GlcNAc modification of some proteins can not be fully excluded. Importantly, azaserine, a widely used GFAT inhibitor not only inhibits HBP pathway under high glucose condition, but also exerts anti-oxidant and anti-inflammatory effects in the endothelial cells under normoglycemic condition in response to TNF $\alpha$, when no increase in HBP flux occurs. ${ }^{12}$ The results implicate that azaserine exhibits anti-oxidative and anti-inflammatory effects in endothelial cells independently of inhibition of HBP pathway. These pharmacological effects of azaserine should be taken into account, when the substance is used to analyze the role of the HBP pathway in a biological system.

In the current article, we presented further results showing that incubation of the endothelial cells with glucosamine to augment
$O$-GlcNAc level does not enhance TNF $\alpha$-induced VCAM- 1 and ICAM-1 protein expression. It however inhibits ICAM-1 (not VCAM-1) expression. Glucosamine itself does not have effects on endothelial expression of the adhesion molecules. The results thus provide further evidence that increased $O$-GlcNAc does not seem to play a role in hyperglycemia-induced upregulation of endothelial expression of the adhesion molecules in response to TNF $\alpha$. The results are also in line with numerous experimental and clinical studies showing that glucosamine exhibits antiinflammatory effects and is used to treat several diseases associated with inflammation. ${ }^{9-11}$ The inhibition of ICAM-1 expression by glucosamine seems not mediated by blockade of oxidative stress, since glucosamine itself induces superoxide anion generation and does not inhibit $\mathrm{TNF} \alpha$-induced superoxide production as demonstrated by the DHE fluorescence staining in the endothelial cells. The pro-oxidative properties of glucosamine have also been reported in renal mesangial cells and pancreatic $\beta$-cells. ${ }^{21,22}$ The results implicate that elevation of $O$-GlcNAc may contribute to the oxidative stress associated with hyperglycemia.

In conclusion, the results presented here provide further evidence that HBP pathway does not mediate the enhancing effect of hyperglycemia on the endothelial inflammatory responses to TNF $\alpha$. In contrast, glucosamine may exert certain anti-inflammatory effect in the context of endothelial adhesion molecule expression through enhancing O-GlcNAc level accompanied by pro-oxidative properties. The pro-oxidative and anti-inflammatory effects of glucosamine might explain the paradoxical effects of enhancing $O$-GlcNAc in cardiovascular system reported by aforementioned various studies. Further work should delineate the definite role of HPB pathway in different aspects of cardiovascular functions, e.g., (1) cardiac vs. vascular injury; (2) effects of acute vs. chronic HBP flux; and (3) effects of diabetic vs. non-diabetic activation of HBP pathway.

\section{Materials and Methods}

Materials. D-(+)-glucosamine hydrochloride and chemicals for immunoblotting were purchased from Sigma (Buchs, Switzerland); antibodies against ICAM-1 and VCAM-1 were from Santa Cruz Biotechnology (Munningen, Switzerland); IRDye 800-conjugated affinity purified goat anti-rabbit $\operatorname{IgG} \mathrm{Fc}_{\mathrm{c}}$ was purchased from BioConcept (Allschwil, Switzerland); Alexa fluor 680-conjugated goat anti-mouse $\operatorname{IgG}(\mathrm{H}+\mathrm{L})$ was from Invitrogen; dihydroethidium (DHE) was purchased from Invitrogen (Lucerne, Switzerland). Endothelial cell growth supplement pack was from PromoCell (Allschwil, Switzerland), and all cell culture media and materials were purchased from GIBCO (Lucerne, Switzerland).

Endothelial cell culture. Endothelial cells were isolated from human umbilical veins and characterized as described. ${ }^{12}$ Cells from the second to fourth passages were used. The endothelial cells were cultured in low-glucose (LG, $5.5 \mathrm{mmol} / \mathrm{l}$ ) DMEM supplemented with $5 \%$ fetal calf serum (FCS), endothelial cell growth supplement and $0.6 \mathrm{nmol} / \mathrm{l}$ insulin. To study effects of $O$-GlcNAc on endothelial expression of adhesion molecules, the cells were incubated with $0.1 \mathrm{mmol} / \mathrm{L}$ or $0.5 \mathrm{mmol} / \mathrm{l}$ of glucosamine for four days. The cells were then cultured in the medium containing 
glucosamine supplemented with a lower concentration of FCS (1\%) for five hours followed by the stimulation with TNF $\alpha$ $(1 \mathrm{ng} / \mathrm{ml})$ for additional 24 hours.

ICAM-1 and VCAM-1 protein expression. Cell lysate preparation, $8 \%$ SDS-PAGE and protein transfer from SDS-PAGE gels to an Immobilon-P membrane (Millipore) were performed as described. ${ }^{12}$ The resultant membranes were first incubated with the individual primary antibody at room temperature for two to three hours after blocking with 5\% skimmed milk. The blots were then incubated with a corresponding anti-mouse (Alexa fluor 680 conjugated) or anti-rabbit (IRDye 800 conjugated) secondary antibody $(1: 5,000)$ for two hours, and the quantification of the signals was performed using the Odyssey Application Software 1.2.

DHE staining for superoxide anion generation. Superoxide anion generation was analyzed as described. ${ }^{12}$ Images were obtained with Zeiss fluorescence microscopy. The intensity of the fluorescence was quantified by Image J software.

Statistical analysis. All data were given as means \pm SE. The ANOVA with Bonferroni post hoc test was used for statistical analysis. A two-tailed value of $\mathrm{p}<0.05$ was considered to indicate a statistically significant difference.

\section{Acknowledgements}

We would like to thank Jean Ruffieux for technical assistance in isolation of endothelial cells. This study was supported by Swiss National Science Foundation (Nr. 3100A0-105917 and 310000120435/1), Swiss Heart Foundation, and the Swiss Cardiovascular Research and Training Network (SCRTN) Programme. J.M.C. was partially supported by Roche Research Foundation.

\section{References}

1. Laczy B, Hill BG, Wang K, Paterson AJ, White CR, Xing D, et al. Protein O-GlcNAcylation: A new signaling paradigm for the cardiovascular system. Am J Physiol Heart Circ Physiol 2009; 296:13-28.

2. Yang X, Ongusaha PP, Miles PD, Havstad JC, Zhang F, So WV, et al. Phosphoinositide signalling links O-GlcNAc transferase to insulin resistance. Nature 2008; 451:964-9.

3. Copeland RJ, Bullen JW, Hart GW. Cross-talk between GlcNAcylation and phosphorylation: Roles in insulin resistance and glucose toxicity. Am J Physiol Endocrinol Metab 2008; 295:17-28.

4. Ngoh GA, Facundo HT, Hamid T, Dillmann W, Zachara NE, Jones SP. Unique hexosaminidase reduces metabolic survival signal and sensitizes cardiac myocytes to hypoxia/ reoxygenation injury. Circ Res 2009; 104:41-9.

5. Champattanachai V, Marchase RB, Chatham JC. Glucosamine protects neonatal cardiomyocytes from ischemia-reperfusion injury via increased protein O-GlcNAc and increased mitochondrial Bcl-2. Am J Physiol Cell Physiol 2008; 294:1509-20.

6. Jones SP, Zachara NE, Ngoh GA, Hill BG, Teshima Y, Bhatnagar A, et al. Cardioprotection by $\mathrm{N}$-acetylglucosamine linkage to cellular proteins. Circulation 2008; 117:1172-82.

7. Fulop N, Zhang Z, Marchase RB, Chatham JC. Glucosamine cardioprotection in perfused rat hearts associated with increased $\mathrm{O}$-linked $\mathrm{N}$-acetylglucosamine protein modification and altered p38 activation. Am J Physiol Heart Circ Physiol 2007; 292:2227-36.

8. Marshall S, Nadeau O, Yamasaki K. Dynamic actions of glucose and glucosamine on hexosamine biosynthesis in isolated adipocytes: Differential effects on glucosamine 6-phosphate, UDP-N-acetylglucosamine and ATP levels. J Biol Chem 2004; 279:35313-9.

9. Zou L, Yang S, Champattanachai V, Hu S, Chaudry IH, Marchase RB, Chatham JC. Glucosamine improves cardiac function following trauma-hemorrhage by increased protein O-GlcNAcylation and attenuation of NF\{kappa\}B signaling. Am J Physiol Heart Circ Physiol 2009; 296:515-23.

10. Ma L, Rudert WA, Harnaha J, Wright M, Machen J, Lakomy R, et al. Immunosuppressive effects of glucosamine. J Biol Chem 2002; 277:39343-9.

11. Xing D, Feng W, Not LG, Miller AP, Zhang Y, Chen YF, et al. Increased protein O-GlcNAc modification inhibits inflammatory and neointimal responses to acute endoluminal arterial injury. Am J Physiol Heart Circ Physiol 2008; 295:335-42.

12. Rajapakse AG, Ming XF, Carvas JM, Yang Z. The hexosamine biosynthesis inhibitor azaserine prevents endothelial inflammation and dysfunction under hyperglycemic condition through antioxidant effects. Am J Physiol Heart Circ Physiol 2009; 296:815-22.
13. Hart GW, Housley MP, Slawson C. Cycling of O-linked $\beta$-N-acetylglucosamine on nucleocytoplasmic proteins. Nature 2007; 446:1017-22.

14. Srinivasan V, Sandhya N, Sampathkumar R, Faroog S, Mohan V, Balasubramanyam M. Glutamine fructose-6-phosphate amidotransferase (GFAT) gene expression and activity in patients with type 2 diabetes: Inter-relationships with hyperglycaemia and oxidative stress. Clin Biochem 2007; 40:952-7.

15. Du XL, Edelstein D, Dimmeler S, Ju O, Sui C, Brownlee M. Hyperglycemia inhibits endothelial nitric oxide synthase activity by posttranslational modification at the Akt site. J Clin Invest 2001; 108:1341-8.

16. Federici M, Menghini R, Mauriello A, Hribal ML, Ferrelli F, Lauro D, et al. Insulindependent activation of endothelial nitric oxide synthase is impaired by O-linked glycosylation modification of signaling proteins in human coronary endothelial cells. Circulation 2002; 106:466-72.

17. Musicki B, Kramer MF, Becker RE, Burnett AL. Inactivation of phosphorylated endothelial nitric oxide synthase (Ser-1177) by O-GlcNAc in diabetes-associated erectile dysfunction. Proc Natl Acad Sci USA 2005; 102:11870-5.

18. Yang Z, Ming XF. Recent advances in understanding endothelial dysfunction in atherosclerosis. Clin Med Res 2006; 4:53-65.

19. Goldberg HJ, Whiteside CI, Hart GW, Fantus IG. Posttranslational, reversible O-glycosylation is stimulated by high glucose and mediates plasminogen activator inhibitor-1 gene expression and Spl transcriptional activity in glomerular mesangial cells. Endocrinology 2006; 147:222-31.

20. Luo B, Soesanto Y, McClain DA. Protein modification by O-linked GlcNAc reduces angiogenesis by inhibiting Akt activity in endothelial cells. Arterioscler Thromb Vasc Biol 2008; 28:651-7.

21. Singh LP, Cheng DW, Kowluru R, Levi E, Jiang Y. Hexosamine induction of oxidative stress, hypertrophy and laminin expression in renal mesangial cells: effect of the antioxidant alpha-lipoic acid. Cell Biochem Funct 2007; 25:537-50.

22. Kaneto H, Xu G, Song KH, Suzuma K, Bonner-Weir S, Sharma A, Weir GC. Activation of the hexosamine pathway leads to deterioration of pancreatic beta-cell function through the induction of oxidative stress. J Biol Chem 2001; 276:31099-104. 


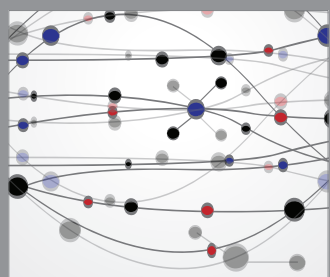

The Scientific World Journal
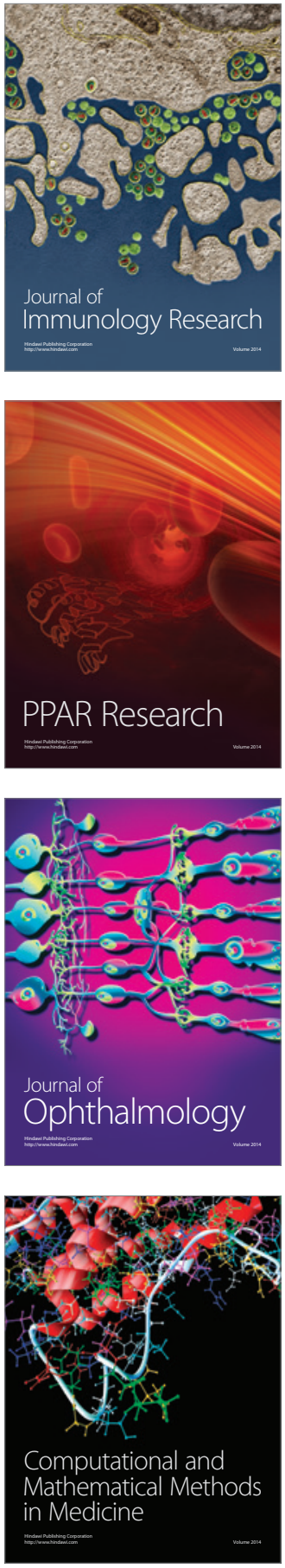

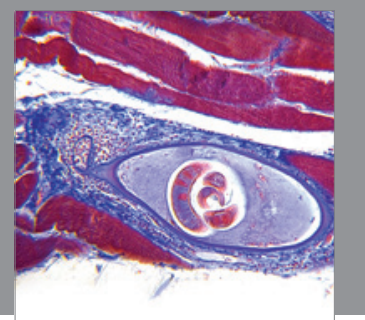

Gastroenterology

Research and Practice
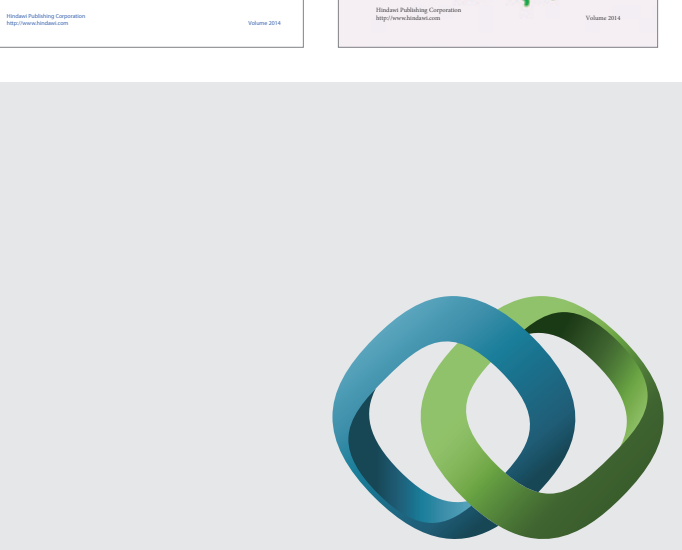

\section{Hindawi}

Submit your manuscripts at

http://www.hindawi.com
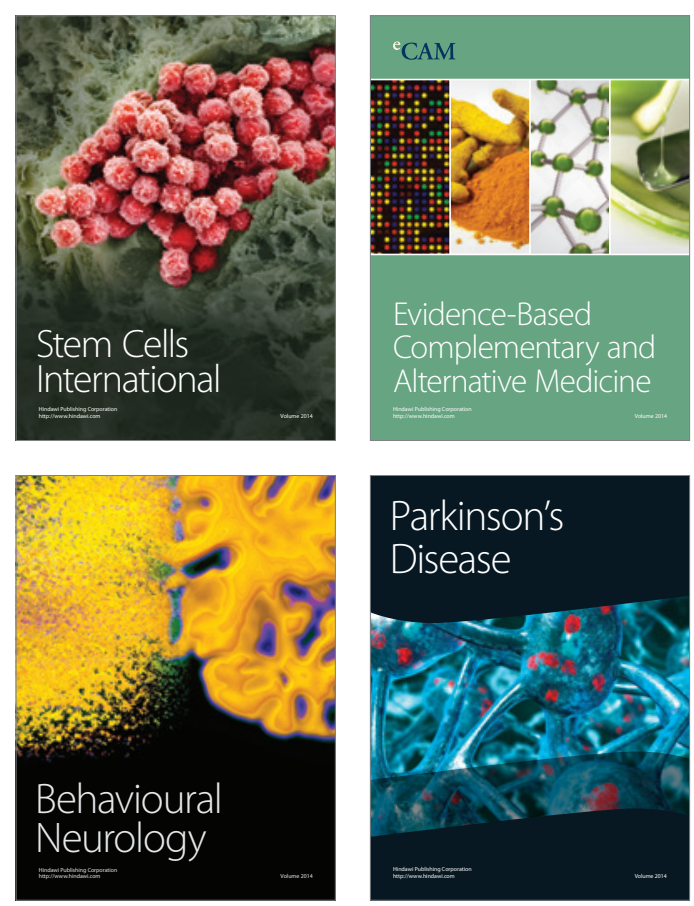

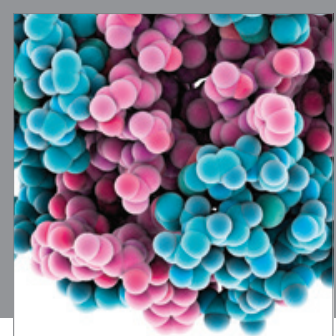

Journal of
Diabetes Research

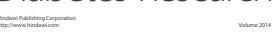

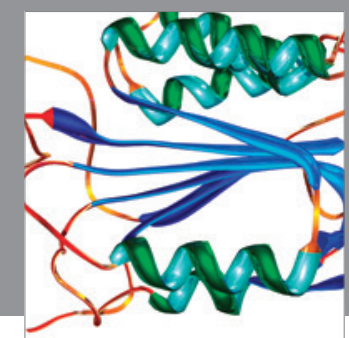

Disease Markers
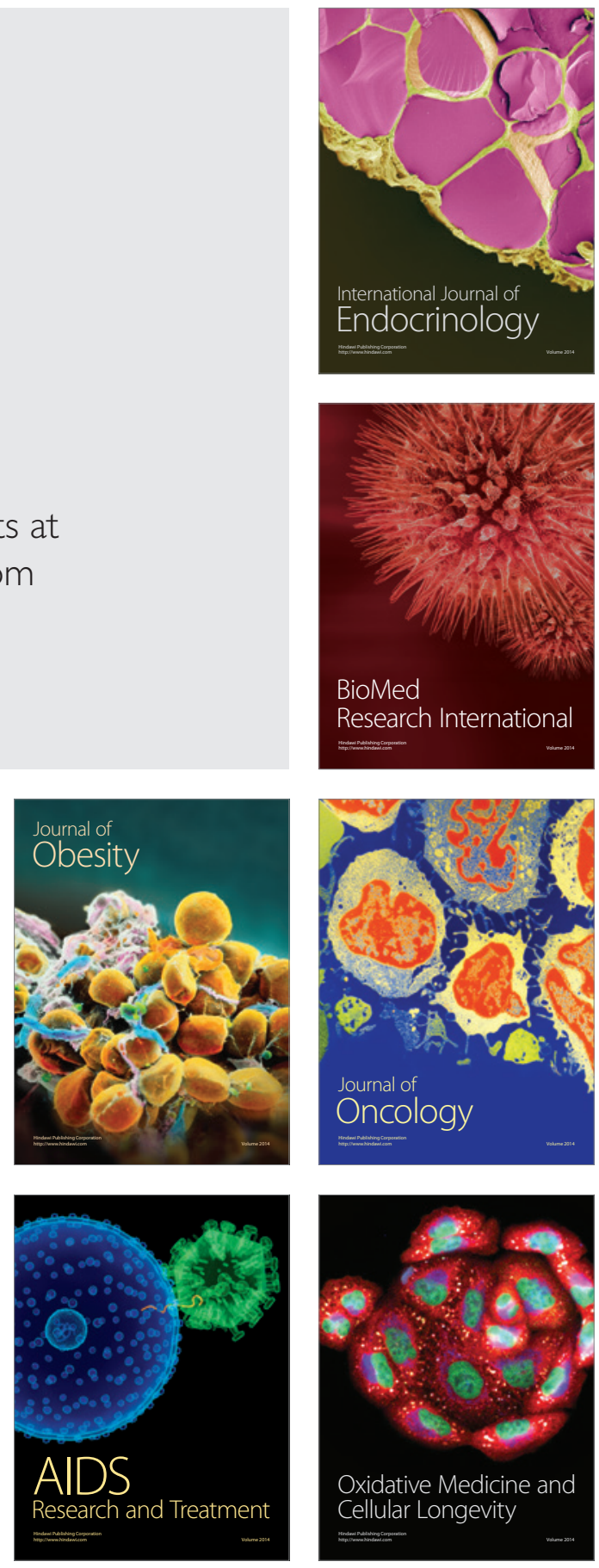